\title{
CrAssphage as a Novel Tool to Detect Human Fecal Contamination on Environmental Surfaces and Hands
}

\author{
Geun Woo Park, Terry Fei Fan Ng, Amy L. Freeland, Vincent C. Marconi, Julie A. Boom, \\ Mary A. Staat, Anna Maria Montmayeur, Hannah Browne, Jothikumar Narayanan, \\ Daniel C. Payne, Cristina V. Cardemil, Aimee Treffiletti, Jan Vinjé
}

\begin{abstract}
CrAssphage is a recently discovered human gut-associated bacteriophage. To validate the potential use of crAssphage for detecting human fecal contamination on environmental surfaces and hands, we tested stool samples $(n=60)$, hand samples $(n=30)$, and environmental swab samples $(n=201)$ from 17 norovirus outbreaks for crAssphage by real-time PCR. In addition, we tested stool samples from healthy persons $(n=173)$, respiratory samples $(n=113)$, and animal fecal specimens $(n=68)$ and further sequenced positive samples. Overall, we detected crAssphage in $71.4 \%$ of outbreak stool samples, $48 \%-68.5 \%$ of stool samples from healthy persons, $56.2 \%$ of environmental swabs, and $60 \%$ of hand rinse samples, but not in human respiratory samples or animal fecal samples. CrAssphage sequences could be grouped into 2 major genetic clusters. Our data suggest that crAssphage could be used to detect human fecal contamination on environmental surfaces and hands.
\end{abstract}

$\mathrm{H}$ ygienic practices, including disinfection of environmental surfaces, are important to reduce exposure to pathogens that spread through fecaloral transmission. Thus, monitoring of human fecal

Author affiliations: Centers for Disease Control and Prevention, Atlanta, Georgia, USA (G.W. Park, T.F.F. Ng, A.L. Freeland,

J. Narayanan, D.C. Payne, C.V. Cardemil, A. Treffiletti, J. Vinjé);

Atlanta Veteran Administration Medical Center, Atlanta

(V.C. Marconi); Emory University School of Medicine, Atlanta

(V.C. Marconi); Texas Children's Hospital, Houston, Texas, USA

(J.A. Boom); Cincinnati Children's Hospital Medical Center,

Cincinnati, Ohio, USA (M.A. Staat); Cherokee Nation Assurance, Arlington, Virginia, USA (A.M. Montmayeur); Oak Ridge Institute for Science and Education, Oak Ridge, Tennessee, USA (H. Browne)

DOI: https://doi.org/10.3201/eid2608.200346 contamination and identifying the source of contamination is an important approach to prevent transmission of gastroenteritis viruses for which humans are the only natural host (e.g., human norovirus) (1). Culturable bacteria (e.g., Escherichia coli, Enterococcus spp., and Bacteroides spp.) are widely used as indicators to assess the presence of human fecal contamination of environmental waters (2-5). However, fecal indicator bacteria are not specific to human fecal contamination (6) and have a poor correlation with exposure risk to enteric viruses (4,7-9).

Over the past few decades, several viruses (e.g., human polyomavirus, Aichi virus, norovirus, and human adenovirus) have been studied as human fecal indicators for the detection of sewage-contaminated source and drinking water (10-13). Recently, both norovirus and adenovirus have been suggested as potential biomarkers of viral contamination to assess hygiene status and potential human health risk of contaminated surfaces and hands of affected persons $(4,12,14-17)$. However, the detection of those viruses in indoor environments was relatively rare and inconsistent, making it difficult to estimate indoor hygiene and limiting their applicability for use in both industrial and regulatory settings $(12,14-17)$.

Recently, a new DNA bacteriophage was discovered by computational analysis of publicly accessible human fecal metagenomics data and was named crAssphage, referring to the Cross-Assembly software that was used for its discovery (18). The single-stranded circular DNA genome is $97 \mathrm{kbp}$ in size with 80 predicted open reading frames (ORFs) (18). Genetically, crAssphage are extremely heterogenous and can be grouped into at least 10 different genera $(18,19)$. Various bacteria of the phylum Bacteroidetes have been proposed as the primary hosts of 
crAssphage, which was supported by recent findings that phage $Ф$ CrAss001 from human feces could be isolated in Bacteroides (20). To date, crAssphage has primarily been detected in human stools and rarely in animals $(18,21)$. In addition, crAssphage can be found at high levels in sewage throughout the year and correlate with the detection of fecal indicators $(E$. coli, enterococcus, human polyomavirus, and somatic coliphage), suggesting they could be used for monitoring human fecal pollution of water (21-25).

In this study, we aimed to validate the potential use of crAssphage to detect human fecal contamination on environmental surfaces and hands. We tested human stool samples, environmental swab samples, and hand rinse samples collected during norovirus outbreaks, as well as stool samples from persons without acute gastroenteritis (AGE) and saliva and nasal samples from humans with respiratory symptoms. To confirm the specificity of crAssphage for the human gut, we also tested fecal specimens from cats, rats, rhesus monkeys, and husbandry animals (cows, pigs, sheep, and horses).

\section{Materials and Methods}

\section{Clinical Samples from Humans}

In this study, we used archived fecal specimens that had been collected from patients with AGE from 5 norovirus outbreaks on cruise ships during 2015-2016 $(\mathrm{n}=30)$ and from 12 norovirus outbreaks in long-term care facilities (LTCFs) in Oregon during 2013-2016 (n = 30) (26). In addition, we tested 22 vomitus samples from norovirus-positive patients as well as 43 saliva and 48 nasal swab samples from norovirus-negative patients. In addition, stool specimens from 2 cohorts of persons without AGE symptoms were included: adults $\geq 25$ years of age who participated as non-AGE controls in a study to determine the incidence of norovirus in Veteran Affairs Medical Centers (VAMCs) (IRB approval no. 00091065) (27) and children $\leq 5$ years of age who participated in the New Vaccine and Surveillance Network (IRB approval no. 6164) (28). Because stool samples from the norovirus outbreaks were categorized as public health nonresearch, human subject regulations did not apply.

\section{Fecal Samples from Animals}

We obtained fecal samples from 3 laboratory animals (rhesus monkeys [ $\mathrm{n}=12]$, rats $[\mathrm{n}=4]$, and cats [ $\mathrm{n}=$ 2]) without diarrhea from archived collections at the Centers for Disease Control and Prevention (CDC) in Atlanta, Georgia. In addition, fecal DNA extracts from cows $(n=10)$, sheep $(n=10)$, horses $(n=10)$, and pigs $(\mathrm{n}=30$ ) were provided by Jeong Kwang Cheol at the University of Florida and Qiuhong Wang at Ohio State University.

\section{Environmental Swab and Hand Rinse Samples}

We collected environmental swab samples at the time of disembarkation of 5 cruise ships (cruise ship A-E) that experienced norovirus outbreaks. We sampled hard surfaces, including toilet seats, toilet door handles, telephone handles, television remote controls, and door handles in cabins that had been occupied by passengers who had reported AGE symptoms by using macrofoam swabs (Puritan, https:/ / www. puritan medproducts.com) as described previously (29). On cruise ship B, we sampled the same environmental surfaces again 3 weeks later when no elevation of the number of AGE cases was reported; these samples are expressed as "B, follow up." We collected all swab samples before standard surface cleaning procedures for each ship. We shipped swab samples on dry ice to $\mathrm{CDC}$ and stored them at $-80^{\circ} \mathrm{C}$ until testing. We also included in this study archived hand rinse samples collected from 30 norovirus patients in LTCFs (26).

\section{Nucleic Acid Extraction and Real-Time PCR Detection of CrAssphage}

We extracted total nucleic acid from clinical samples, hand rinse samples, and swabs, as described previously (26,29; Appendix, https://wwwnc.cdc.gov/ EID/article/26/8/20-0346-App1.pdf). We designed oligonucleotide primers and probes on the basis of conserved regions of the DNA polymerase gene (ORF00018) from 43 publicly available crAssphage strains by using the real-time quantitative PCR assay tool from Integrated DNA Technologies (https:/ / www.idtdna.com) (18). Primer sequences had no more than 1 mismatch with the prototype CrAssphage genome (GenBank accession no. NC_024711). We tested extracted DNA from clinical and environmental samples by using TaqMan real-time PCR and the AgPath-ID One Step RT-PCR Kit (ThermoFisher Scientific, https://www.thermofisher.com ) on an Applied Biosystems 7500 platform (also ThermoFisher Scientific); the oligonucleotide primers (TN201/ TN203) and probe (TN202) generated a 146-bp product (Table 1) (Appendix). We amplified the full-length ORF00018 (DNA polymerase) gene of a sample (ship $\mathrm{E}$ [stool II] in Figure 1) by using primers CrAssPol-F and CrAsspol-R (Table 1) to generate a 2,428-bp amplicon, as described previously (30). In each experiment, we included a 10 -fold serial dilution $\left(10^{5.7}-10^{0.7}\right.$ copies $/ 3 \mu \mathrm{L}$ ) of this purified and quantified amplicon to generate a standard curve. The detection limit of 
Table 1. Oligonucleotide primers and probe used for detection and typing of crAssphage

\begin{tabular}{|c|c|c|c|}
\hline $\begin{array}{l}\text { Primer or } \\
\text { probe }\end{array}$ & DNA sequence & Position* & Description \\
\hline CrAssPol_F† & 5'-CGG CGG GTT AAT CAA AAT AGA A-3' & $\begin{array}{c}8907-8928 \\
\text { (flanking pol) }\end{array}$ & $\begin{array}{c}\text { Forward primer } \\
\text { conventional PCR }\end{array}$ \\
\hline CrAssPol_R† & 5'-GCG GAG AAC CCC ATT TAT TAA TAA G-3' & $\begin{array}{l}\text { 11334-11310 } \\
\text { (flanking pol) }\end{array}$ & $\begin{array}{c}\text { Reverse primer } \\
\text { conventional PCR }\end{array}$ \\
\hline TN201 & 5'-ATG TWG GTA RAC AAT TTC ATG TAG AAG-3' & $\begin{array}{l}\text { 10919-10945 } \\
\text { (within pol) }\end{array}$ & $\begin{array}{c}\text { Forward primer } \\
\text { real-time PCR }\end{array}$ \\
\hline TN203 & 5'-TCA TCA AGA CTA TTA ATA ACD GTN ACA ACA-3' & $\begin{array}{c}\text { 11111-11082 } \\
\text { (within pol) }\end{array}$ & $\begin{array}{c}\text { Reverse primer real-time } \\
\text { PCR and typing PCR }\end{array}$ \\
\hline TN202 & FAM-5-ACC AGC MGC CAT TCT ACT ACG AGH AC-3-BHQ1 & $\begin{array}{c}\text { 11079-11054 } \\
\text { (within pol) }\end{array}$ & Probe real-time PCR \\
\hline JP1crasF & 5'-TAA AAC TAC WAT TTA TAG AGT TAA TAA AGA TGC STT TAG T-3' & $\begin{array}{c}10023-10062 \\
\text { (within pol) }\end{array}$ & Forward primer typing \\
\hline
\end{tabular}

the crAssphage real-time PCR was 0.7 DNA copies per PCR reaction. The average slope and deviation of the standard curves was $3.3626 \pm 0.0377\left(r^{2} \geq 0.9926\right)$. We calculated the concentration of crAssphage by converting cycle threshold values to DNA copies.

\section{DNA Sequencing of crAssphage}

To enable sequencing, we amplified DNA from realtime PCR-positive samples by using oligonucleotide primers JP1crasF/TN203 (Table 1) to generate a 1,089bp PCR amplicon (Appendix). We sequenced the purified PCR products by using 500-cycle $(2 \times 250$-bp paired-end) MiSeq Reagent Kit (Illumina, https:// www.illumina.com). After filtering and trimming raw sequence reads, we assembled contigs by using the de novo assembler SPAdes 3.7.0 (http://cab.spbu.ru/ software/spades) (Appendix). We analyzed assembled crAssphage amplicon sequences by read mapping and gene annotation using Geneious 11.1.2 (Biomatters, https://www.geneious.com), as described previously (31). The GenBank accession numbers for the strains sequenced in this study are MT475797-824 (Figure 1) and MT475766-96 (Figure 2).

\section{Phylogenetic and Sequence Analyses}

We generated multiple sequence alignment of crAssphage sequences by using MUSCLE (32) and constructed maximum-likelihood phylogenetic trees by using PhyML (33). We used the best nucleotide substitution model analyzed by Smart Model Selection based on

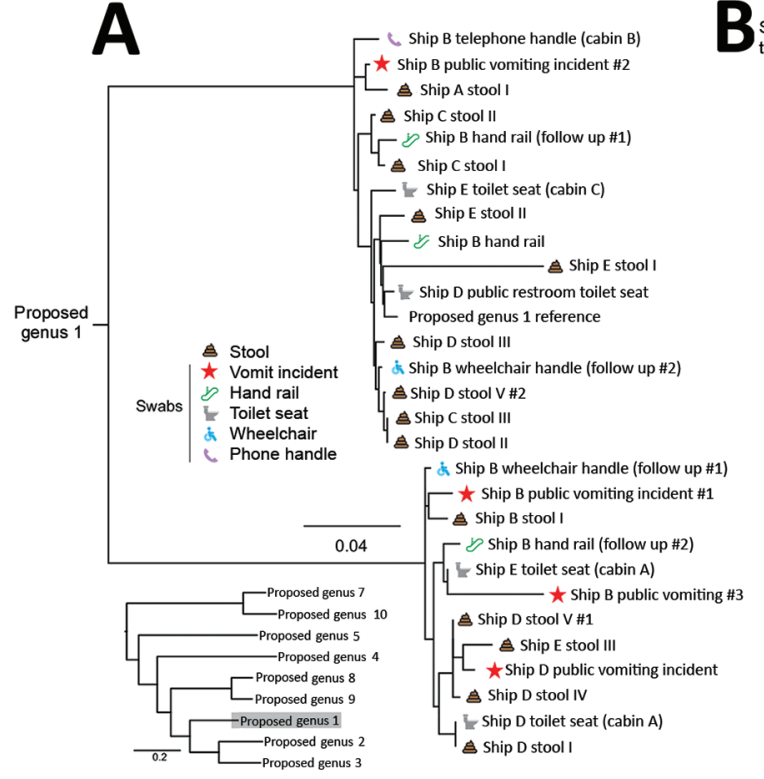

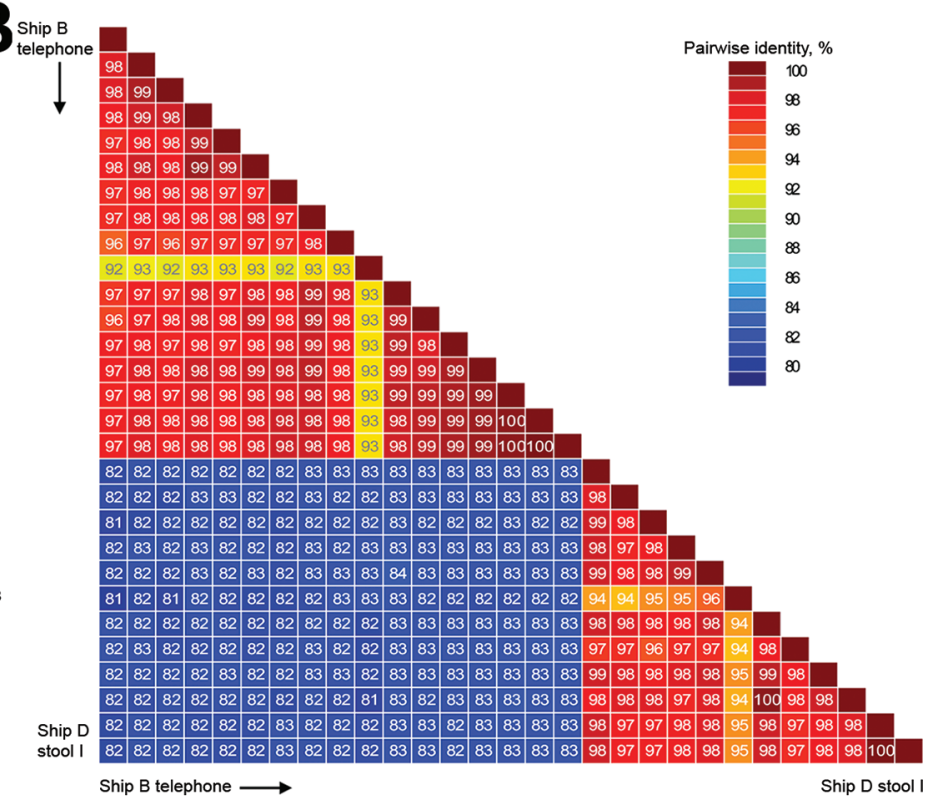

Ship B telephone $\longrightarrow$
Ship D stool I

Figure 1. Phylogenetic relationships and pairwise sequence comparison of crAssphage strains from swab samples collected during norovirus outbreaks on cruise ships. A) Phylogeny of crAssphage on cruise ships, showing ship and source for each strain. Inset shows position of cruise ship strains among reference strains; scale bar indicates number of nucleotide changes between sequences. B) Colorcoded pairwise identity matrix for crAssphage strains. Each cell includes the percentage identity among 2 sequences (horizontally to the left and vertically at the bottom). Key indicates pairwise identity percentages. 

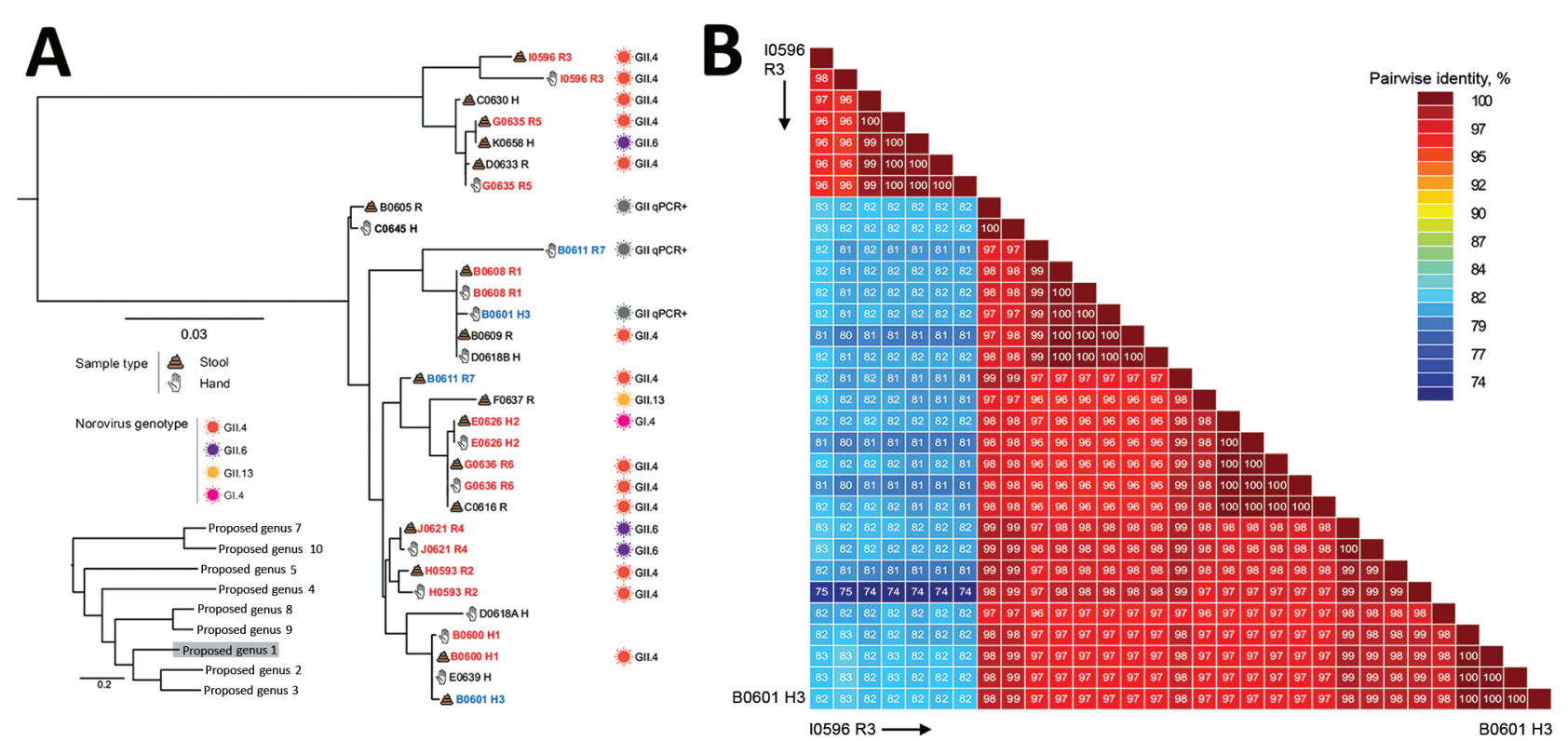

Figure 2. Phylogenetic relationships and pairwise sequence comparison of crAssphage strains from hand rinse samples collected during norovirus outbreaks at long-term care facilities. A) Phylogeny of crAssphage strains. Strain identification includes facility (A-J), strain number, and human source $(\mathrm{H}$, healthcare worker; $\mathrm{R}$, resident) for each isolate. Sample source and genotypes are indicated. Red strain names indicate that both hand and stool sample are genetically related, blue strain names that paired hand and stool samples are genetically distinct. Black strain names indicate hand or stool sample pairs that tested negative for crAssphage. Inset shows position of long-term care strains among reference strains; scale bar indicates number of nucleotide changes between sequences. (B) Color-coded pairwise identity matrix for crAssphage strains. Each cell includes the percentage identity among 2 sequences (horizontally to the left and vertically at the bottom). Key indicates pairwise identity percentages

the Bayesian information criterion (34) and calculated pairwise nucleotide identity $(\mathrm{NI})$ between sequences by using the Sequence Demarcation Tool (35).

\section{Data Analysis}

We determined sensitivity, specificity, and predictive values of crAssphage on norovirus co-infection (or cocontamination) as described previously (36). We performed log transformation, followed by the Wilcoxon rank-sum test, to compare the median crAssphage concentration $\left(\log _{10}\right.$ genomic copies per sampled object for surface and hand rinse sample or per gram for stool) between different comparison groups. We used SPSS Statistics 21 (IBM, https:/ / www.ibm.com) for statistical calculations and considered differences with $\mathrm{p}$ values $<0.05$ to be statistically significant (37).

\section{Results}

\section{Detection of crAssphage in Human and Animal Samples}

Overall, we detected crAssphage in $71.4 \%$ of human stool samples, including $42(70.0 \%)$ of the 60 stool samples collected from 17 norovirus outbreaks, 46 $(48 \%)$ of the 96 stool samples from adults $\geq 25$ years of age without AGE symptoms and $53(68.8 \%)$ of the
77 stool samples from children $\leq 5$ years of age with AGE symptoms (Table 2). Specifically, 23 (76.7\%) of the 30 stool samples from the 5 norovirus outbreaks on cruise ships tested positive, and $19(63.3 \%)$ of 30 stool samples collected from the 12 norovirus outbreaks in LTCFs tested positive (Table 2). Thirty-nine $(65.0 \%)$ of the 60 stool samples tested positive for both crAssphage and norovirus. The median concentration of crAssphage per gram of stool ranged from 5.9 (range 2.8-8.9) $\log _{10}$ genome copies in samples from norovirus outbreaks to 8.1 (range 3.1-10.3) $\log _{10}$ genomic copies in samples from adults without AGE symptoms and 8.4 (range 4.1-10.1) $\log _{10}$ genomic copies in samples from children $\leq 5$ years of age without AGE symptoms. All vomitus samples from patients in norovirus outbreaks as well as saliva and nasal swab samples from children with respiratory symptoms tested negative for crAssphage. We did not detect crAssphage in any of the 78 fecal samples from animals.

\section{Detection of crAssphage on Environmental Surfaces on Cruise Ships}

We collected a total of 201 swab samples from frequently touched surfaces on 6 cruise ship voyages (5 cruise ships $[\mathrm{A}-\mathrm{E}])$ during norovirus outbreaks and 
Table 2. Prevalence of crAssphage in stool samples from norovirus outbreaks on cruise ships and in long-term care facilities and healthy controls without acute gastroenteritis

\begin{tabular}{lccc}
\hline Setting (no. samples) & Age, y, (range) & $\begin{array}{c}\text { \% CrAssphage (no. } \\
\text { positive/no. tested) }\end{array}$ & crAssphage titer (range) \\
\hline Cruise ship voyages† (5) & $65.5(29-88)$ & $76.7 \%(23 / 30)$ & $4.5(3.2-8.9)$ \\
Long-term care facilities $\ddagger(12)$ & $63.5(18-87)$ & $63.3 \%(19 / 30)$ & $5.4(2.8-8.9)$ \\
Adults without acute gastroenteritis (96) & $59.0(28-83)$ & $48 \%(46 / 96)$ & $8.1(3.1-10.3)$ \\
Children without acute gastroenteritis (77) & $1.1(0.2-5.0)$ & $68.8 \%(53 / 77)$ & $8.4(4.1-10.1)$ \\
\hline *log10 genomic copies per gram of stool sample. & & & \\
†Tis study. & & & \\
†Park et al. (26). & & & \\
\hline
\end{tabular}

1 cruise ship (B, follow up) 3 weeks after a norovirus outbreak). We detected crAssphage DNA in 113 (56.2\%) of the swab samples. The rates for each individual cruise ship were as follows: $15.2 \%(5 / 33)$ (cruise ship A); 84.8\% (28/33) (cruise ship B); 62.3\% $(19 / 31)$ (cruise ship C); $44.7 \%$ (17/38) (cruise ship D); $72.7 \%$ (24/33) (cruise ship E); and 60.6\% (20/33) (cruise ship (B, follow up).

\section{On Surfaces in Cabins of Norovirus-Positive Patients}

A total of $47(58.8 \%)$ of 80 swab samples tested positive for crAssphage; median concentration was 2.5 (0.8-5.6) $\log _{10}$ genomic copies per surface (Table 3). Remote controls had the highest crAssphage contamination $(87.5 \%)$, followed by toilet seats $(68.5 \%)$, which had the highest crAssphage titer, $3.3 \log _{10}$ (1.2-5.6) $\log _{10}$ genomic copies per seat. Of the $80 \mathrm{swab}$ samples, 29 (36.3\%) tested positive for norovirus; 11 of those samples also tested positive for crAssphage. The positive predictive value (PPV) of norovirus coinfection of crAssphage-positive samples was 38.0\%; the negative predictive value (NPV) was $29.4 \%$ (Table 4). Compared with all surfaces sampled, toilet seats $(56.3 \%)$ in the cabins from AGE-positive passengers had the highest norovirus contamination, with an average concentration of $5.5(3.1-7.4) \log _{10}$ genomic copies per surface.

\section{On Surfaces in Public Areas}

Of all surfaces in public areas on cruise ships that were sampled, $51.5 \%(21 / 64)$ tested positive for crAssphage; median concentration was 2.4 (1.6-3.2) $\log _{10}$ genomic copies per surface. Five surfaces in public areas (casino chips, a medical center clipboard, gift shop register touch screens, surfaces that were exposed to a public vomiting incident, and edges of a trolley for dirty linen) had contamination of $\geq 50 \%$. Only 5 surfaces, including a menu folder (cruise ship A), a medical center clipboard (cruise ship A), hand contact surfaces in the public vomiting incident location (cruise ship A), hand rails in the atrium (cruise ship B), and ATM buttons (cruise ship C), tested positive for human norovirus; titers were 1.8-5.1 $\log _{10}$ genomic copies per surface. Two of the 5 noroviruspositive surfaces (a medical center clipboard and a handrail in the atrium) also tested positive for crAssphage. PPV for norovirus co-contamination of crAssphage-positive surfaces was $40.0 \%$; NPV was 67.8 .

\section{Three Weeks after a Norovirus Outbreak}

Seven $(46.7 \%)$ of the 15 swab samples collected from cabins on cruise ship B that had been occupied by patients with AGE 3 weeks earlier tested positive for crAssphage, whereas 7 (38.9\%) of the 18 surfaces in public areas tested positive. Compared with results from the same cruise ship immediately after the outbreak 3 weeks earlier, the number of crAssphagepositive surfaces decreased from 12 to 7 in public areas of cruise ship B. However, swabs from 6 surfaces (ATM buttons, a buffet utensil, a medical center clipboard, atrium handrails, a wheelchair handle rest in passenger areas, and smoking bar countertop surfaces in the crew smoking room) tested positive for crAssphage again. Two crAssphage-positive swab samples collected from a handrail (ship B handrail, follow-ups 1 and 2) and a wheelchair (ship B wheelchair, follow-ups 1 and 2) on cruise ship B contained multiple crAssphage sequences (Figure 1). These sequences were genetically distinct from those detected during the norovirus outbreak on the same cruise ship 3 weeks earlier (voyage B), suggesting not persistence of previous fecal material but more recent contamination with human fecal matter. In contrast, norovirus contamination on surfaces decreased from $24.2 \%(8 / 33)$ during the outbreak to $3.0 \%(1 / 33) 3$ weeks later.

\section{Contamination of Hands with crAssphage during Norovirus Outbreaks in LTCFs}

In total, $18(60.0 \%)$ of 30 hand rinse samples tested positive for crAssphage, including samples from 7 healthcare workers (HCWs) and 11 residents. Both hand rinse and stool samples from 15 norovirus patients (4 HCWs and 11 residents) tested positive for crAssphage (Appendix Table). In a previous study, we reported that $14(46.7 \%)$ of 30 hand rinse samples 
tested positive for norovirus, including $3 \mathrm{HCWs}$ and 11 residents (26). Overall, $2(28.6 \%)$ of 7 crAssphage-positive hand rinse samples from HCWs and 8 $(72.7 \%)$ of 11 from residents tested positive for human norovirus. The PPV was $72.7 \%$ and the NPV $25.0 \%$ for co-contamination of crAssphage-positive hands of residents with norovirus, whereas for $\mathrm{HCWs}$ the PPV was $66.7 \%$ and NPV $58.3 \%$.

\section{Sequence and Phylogenetic Analysis of crAssphage}

Of the 42 PCR-positive stool samples, 30 (71.4\%) were successfully sequenced and the titer of the remaining 12 samples was too low. Sequences from several crAssphage were identical (e.g., ship Dstool V\# 1 and ship D stool IV, and ship C stool III and ship D stool II) (Figure 1). Overall, crAssphage sequences detected in samples from cruise ships rarely clustered closely together (Figure 1), whereas crAssphage from LTCFs were more closely related, with near-identical sequences (99\%-100\% NI) (Figure 2).

From the cruise ship outbreaks, 13 (56.5\%) of the 23 crAssphage-positive stool samples and 10 (8.8\%) of 113 crAssphage-positive swab samples were sequenced successfully. Phylogenetic analysis showed that crAssphage sequences could be grouped in 2 genetic clusters within proposed genus 1 (19) (Figure 1). Several samples contained multiple crAssphage sequences, including multiple stool samples with $>1$ sequence (ship D), swab samples from a wheelchair and a handrail each containing 2 sequences (ship B, follow up), and a swab sample from a public vomiting event (ship B) containing 3 different crAssphage sequences.

From the crAssphage in LTCFs, 17 (89.5\%) of 19 stool samples and $13(72.2 \%)$ of 18 positive hand rinse samples were successfully sequenced. The sequences could be grouped in 2 clusters that had $<83 \% \mathrm{NI}$ (Figure 2, panel A). Within each cluster, crAssphage was genetically diverse, with pairwise NI ranging from $96 \%$ to $100 \%$ (Figure 2, panel B). Identical crAssphage sequences were detected in paired hand rinse and stool samples from 5 persons ( 3 residents [G0636, B0608, and J0621] and 2 HCWs [E0626 and B0600]) (Figure 2). Among those persons, 2 residents [G0636, and J0621] had identical norovirus sequences in paired hand and stool samples as well. In contrast, crAssphage sequences in stool samples from 2

\begin{tabular}{|c|c|c|c|c|}
\hline \multirow[b]{2}{*}{ Sampled objects† } & \multicolumn{2}{|c|}{ CrAssphage } & \multicolumn{2}{|c|}{ Norovirus } \\
\hline & $\begin{array}{l}\text { No. positive/total } \\
\text { no. }(\%)\end{array}$ & $\begin{array}{l}\text { Concentration* } \\
\text { (range) }\end{array}$ & $\begin{array}{l}\text { No. positive/total } \\
\text { no. }(\%)\end{array}$ & $\begin{array}{l}\text { Concentration* } \\
\text { (range) }\end{array}$ \\
\hline \multicolumn{5}{|l|}{ Cabins of norovirus-positive patients } \\
\hline Toilet seats ${ }^{P}$ & $11 / 16(68.5)$ & $3.3(1.2-5.6)$ & 9/16 (56.3) & $5.5(3.1-7.4)$ \\
\hline Toilet door handles ${ }^{\mathrm{M}}$ & $7 / 16(43.75)$ & $2.3(1.0-3.1)$ & $5 / 16(31.3)$ & $5.1(3.7-5.7)$ \\
\hline Telephone handles $^{P}$ & $9 / 16(56.4)$ & $2.4(1.7-3.5)$ & $3 / 16(18.8)$ & $4.9(4.9-5.5)$ \\
\hline Remote control surfaces ${ }^{P}$ & $14 / 16(87.5)$ & $2.6(1.4-4.1)$ & $5 / 16(31.3)$ & $3.6(2.9-5.1)$ \\
\hline Cabin door handles ${ }^{\mathrm{M}}$ & 6/16 (37.5) & $2.0(0.8-3.4)$ & 6/16 (37.5) & $4.4(3.1-6.0)$ \\
\hline Overall & $47 / 80(58.8)$ & $2.5(0.8-5.6)$ & $29 / 80(36.3)$ & $4.8(3.1-7.4)$ \\
\hline \multicolumn{5}{|l|}{ Public area (passenger area) } \\
\hline ATM buttons $^{P}$ & 2/3 (66.6) & $2.8(2.4-3.4)$ & 1/3 (33.3) & 1.8 \\
\hline Menu folder ${ }^{L}$ & $2 / 5(40)$ & $1.9(1.7-2.1)$ & $1 / 5(20.0)$ & 4.9 \\
\hline Condiment containers ${ }^{\mathrm{M}}$ & $2 / 5(40)$ & $2.2(2.1-2.3)$ & $0 / 5(0)$ & \\
\hline Buffet utensils $^{M}$ & $1 / 2(50)$ & 1.6 & $0 / 2(0)$ & \\
\hline Ice cream handle $P$ & $1 / 4(25.0)$ & 2.2 & $0 / 3(0)$ & \\
\hline Casino chips $^{P}$ & $3 / 5(60)$ & $2.7(2.1-2.8)$ & $0 / 5(0)$ & \\
\hline Medical center clipboards ${ }^{P}$ & $3 / 5(60)$ & $2.3(2.1-2.5)$ & $1 / 5(20)$ & 5.1 \\
\hline Gift shop register touch screens ${ }^{P}$ & $3 / 5(60)$ & $3.0(2.7-3.0)$ & $0 / 5(0)$ & \\
\hline Youth center toys ${ }^{P}$ & $2 / 4(50.0)$ & $2.1(2.1-2.2)$ & $0 / 4(0)$ & \\
\hline Atrium hand rails $\mathrm{W}$ & $2 / 5(40.0)$ & $3.0(2.1-3.9)$ & $1 / 5(20)$ & 4.1 \\
\hline Internet café keyboards ${ }^{P}$ & $3 / 5(60)$ & $1.8(1.8-2.5)$ & $0 / 5(0)$ & \\
\hline Wheelchair handle rests ${ }^{P}$ & $2 / 5(25)$ & $2.9(2.0-3.8)$ & $0 / 5(0)$ & \\
\hline $\begin{array}{l}\text { Hand contact surfaces exposed to public } \\
\text { vomiting incident }{ }^{\mathrm{M}}\end{array}$ & $2 / 4(50)$ & $3.2(2.8-3.5)$ & $1 / 4(25)$ & 4.3 \\
\hline Toilet seat surfaces in public rest room ${ }^{P}$ & $3 / 3(100)$ & $4.7(2.4-4.7)$ & $0 / 3(0)$ & 0 \\
\hline Overall & $21 / 64(51.5)$ & $2.4(1.6-3.2)$ & $5 / 64(7.8)$ & $3.3(1.8-5.1)$ \\
\hline \multicolumn{5}{|l|}{ Public area (crew area) } \\
\hline Time clock machines $^{P}$ & $3 / 5(60)$ & $2.2(2.1-2.5)$ & $0 / 5(0)$ & \\
\hline Edges of trolley for dirty linens ${ }^{P}$ & $5 / 5(100)$ & $2.3(1.8-3.2)$ & $0 / 5(0)$ & \\
\hline Elevator buttons in food service areas ${ }^{P}$ & $3 / 5(60)$ & $2.3(2.1-3.1)$ & $0 / 5(0)$ & \\
\hline Computer keyboard $^{\mathrm{P}}$ & $2 / 5(40)$ & $2.3(2.0-2.5)$ & $0 / 5(0)$ & \\
\hline Countertop surfaces in crew smoking roomw & $2 / 5(40)$ & $2.3(2.1-2.5)$ & $0 / 5(0)$ & \\
\hline Overall & $15 / 25(60)$ & $2.3(1.8-3.2)$ & $0 / 25(0)$ & \\
\hline
\end{tabular}

${ }^{1} \log _{10}$ genomic copies per sampled object.

${ }^{2}$ Each superscripted character indicates surface material of sampled object as follows: P, plastic; M, metal; W, wood; and L, leather. 
Table 4. Sensitivity, specificity, and predictive values of norovirus co-contamination on crAssphage positive environmental surfaces and hand rinse samples*

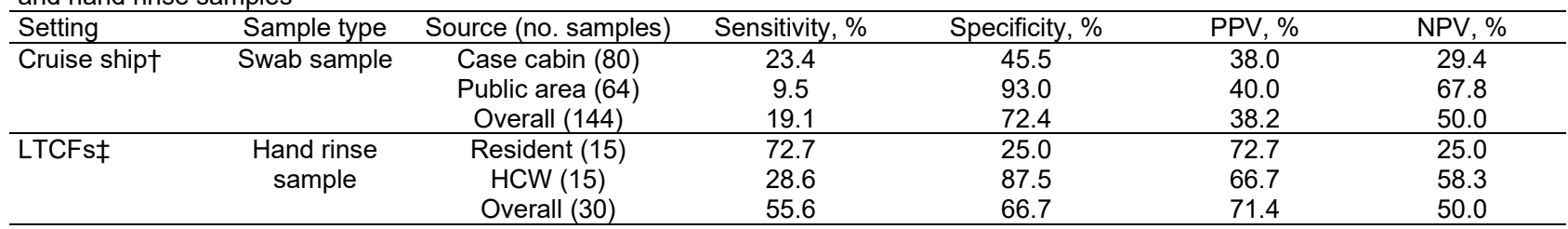

${ }^{*} \mathrm{HCW}$, healthcare worker; LTCF, long-term care facility; NPV, negative predictive value; PPV, positive predictive value.

†This study

$\ddagger$ Park et al. (26).

patients (resident D0611 and HCW B0601) did not match their corresponding hand rinse sample. Also, norovirus was detected only in the hand sample, not a stool sample, from HCW B0601. A single crAssphage sequence was detected on hands from $2 \mathrm{HCWs}$ (D0645 and C0639), and 2 genetically different crAssphages were detected on the hand from $1 \mathrm{HCW}$ (D0618), whereas all stool samples from these $3 \mathrm{HCW}$ tested crAssphage negative.

The median concentration of crAssphage in the 30 stool samples that could be sequenced was 6.5 (range 2.8-8.9) $\log _{10}$ genomic copies per gram of stool, compared with 3.9 (range 3.2-6.6) copies for samples that could not be sequenced $(p<0.001)$. Viral load of crAssphage detected in environmental samples that could be sequenced was $3.5(2.0-5.4) \log _{10}$ genomic copies $(\mathrm{n}=10)$, compared with 2.3 (range 0.6-5.6) copies $(\mathrm{n}=$ 103) for samples that could not be sequenced.

\section{Discussion}

We detected crAssphage in $>60 \%$ of stool samples from patients with AGE during norovirus outbreaks as well in at least half of stool samples from healthy populations but not in other clinical materials (vomitus, saliva, or nasal rinse) or fecal specimens from animals. The high prevalence of crAssphage on surfaces and hands in norovirus outbreak settings suggests that these phages can be used as an indicator to monitor human fecal contamination of environmental sources other than sewage-contaminated water (21-25). CrAssphage contamination was also frequently found on environmental surfaces in public areas of cruise ships both during and after norovirus outbreaks, suggesting a potential role of crAssphage in monitoring fecal contamination on surfaces in common settings that could be targeted for enhanced cleaning and disinfection practices.

CrAssphage can be classified into 4 subfamilies, which can be further divided into 10 candidate genera $(18,19)$. In agreement with data from previous studies $(19,38)$, we found genetically different phages in stools and environmental surfaces from different norovirus outbreaks. The extreme genetic diversity of crAssphage could help to determine possible contamination sources. For example, crAssphage strains in stool samples collected from the same LTCFs during outbreaks displayed a strong degree of interpersonal variation. Thus, identical crAssphage sequences found in stool and hand rinse samples of the same person suggest self-contamination, whereas different sequences suggest possible contamination with fecal material from someone else. On the basis of these assumptions, we concluded that the hands of most LTCF residents were frequently self-contaminated, whereas the hands of HCWs were more likely cross-contaminated, either by contact with frequently touched environmental surfaces or by assisting norovirus patients, highlighting the need to strictly adhere to hand hygiene practices and to take additional contact precautionary measures during norovirus outbreaks.

This study has several limitations. First, although we designed our PCR assay to detect crAssphage based on a larger number of sequences than were used in previous studies $(21,23,30,39)$, only viruses from 1 of the 10 recognized crAssphage genera were detected, suggesting that crAssphage from other genera would likely have been missed. Second, because gastroenteritis viruses such as norovirus are often transmitted through vomitus or aerosols, use of crAssphage during outbreaks might be limited (40-42). Finally, because crAssphage assay was not validated with other domestic animals that share human-occupied spaces (e.g., dogs), nonhuman fecal contamination could not completely be ruled out.

CrAssphage are strongly correlated with bacterial species related to Bacteroidetes but are not associated with diarrheal disease in adults $(38,43)$. Thus, the presence of crAssphage does not correlate with norovirus contamination but rather with human fecal contamination. Detection of crAssphage on environmental surfaces might help to better assess exposure risk for human norovirus in public areas (e.g., on cruise ships) as well as help to identify 
frequently touched surfaces that are often fecally contaminated as key sites for enhanced cleaning practices. Sequence analysis of crAssphage in paired hand rinse and stool samples provided laboratory evidence that hands of several persons were likely cross-contaminated with fecal material from other patients, suggesting that crAssphage can be used as a tool to monitor fecal contamination patterns. Because we did not test crAssphage contamination before or after cleaning of environmental surfaces, or assess hand hygiene practices performed by the staff in the LTCFs during the norovirus outbreaks, we recommend additional studies to guide prevention measures, such as enhanced cleaning (e.g., use of the US Environmental Protection Agency's registered products of list $G$ ).

\section{Acknowledgments}

We thank Suxiang Tong, Sungsil Moon, and Yuhuan Wang, Peixin Fan, Kwang Cheol, and Jeong Qiuhong Wang for providing human clinical samples or animal fecal (or DNA) samples. We also thank Matthew Jaqua, John Oh, Rachele Cruz, Sandra Martin, Keenan Williamson, Catherine Yen, and Elizabeth De Nardo for coordinating enrollment and data/sample collection during the norovirus outbreaks in long-term care facilities; LaDonna Grenz, Marjorie Yungclass, Laura Tsaknaridis, and Vanda Makris for assistance with testing and shipping of samples; and Vincent R. Hill for critical reading the manuscript.

\section{About the Author}

Dr. Park is a microbiologist at the National Calicivirus Laboratory in the Division of Viral Diseases, National Center for Immunization and Respiratory Diseases, Centers for Disease Control and Prevention, Atlanta, Georgia. His research interests include environmental surveillance and disinfection of noroviruses and other viral gastroenteritis viruses.

\section{References}

1. Bányai K, Estes MK, Martella V, Parashar UD. Viral gastroenteritis. Lancet. 2018;392:175-86. https:// doi.org/ 10.1016/S0140-6736(18)31128-0

2. Harwood VJ, Staley C, Badgley BD, Borges K, Korajkic A. Microbial source tracking markers for detection of fecal contamination in environmental waters: relationships between pathogens and human health outcomes. FEMS Microbiol Rev. 2014;38:1-40. https://doi.org/10.1111/ 1574-6976.12031

3. Julian TR, MacDonald LH, Guo Y, Marks SJ, Kosek M, Yori PP, et al. Fecal indicator bacteria contamination of fomites and household demand for surface disinfection products: a case study from Peru. Am J Trop
Med Hyg. 2013;89:869-72. https:// doi.org/10.4269/ ajtmh.12-0425

4. Mattioli MC, Davis J, Mrisho M, Boehm AB. Quantification of human norovirus GII on hands of mothers with children under the age of five years in Bagamoyo, Tanzania. Am J Trop Med Hyg. 2015;93:478-84. https:/ / doi.org/ 10.4269/ajtmh.14-0778

5. Fiksdal L, Maki JS, LaCroix SJ, Staley JT. Survival and detection of Bacteroides spp., prospective indicator bacteria. Appl Environ Microbiol. 1985;49:148-50. https://doi.org/10.1128/AEM.49.1.148-150.1985

6. Sinton LW, Finlay RK, Hannah DJ. Distinguishing human from animal faecal contamination in water: a review. N Z J Mar Freshw Res. 1998;32:323-48. https:/ / doi.org/10.1080/ 00288330.1998 .9516828

7. Haramoto E, Kitajima M, Hata A, Torrey JR, Masago Y, Sano D, et al. A review on recent progress in the detection methods and prevalence of human enteric viruses in water. Water Res. 2018;135:168-86. https:/ / doi.org/10.1016/ j.watres.2018.02.004

8. Malla B, Ghaju Shrestha R, Tandukar S, Sherchand JB, Haramoto E. Performance evaluation of human-specific viral markers and application of pepper mild mottle virus and CrAssphage to environmental water samples as fecal pollution markers in the Kathmandu Valley, Nepal. Food Environ Virol. 2019;11:274-87. https:/ / doi.org/10.1007/ s12560-019-09389-x

9. De Giglio O, Caggiano G, Bagordo F, Barbuti G, Brigida S, Lugoli F, et al. Enteric viruses and fecal bacteria indicators to assess groundwater quality and suitability for irrigation. Int J Environ Res Public Health. 2017;14:558. https:/ / doi.org/ 10.3390/ijerph14060558

10. Jiang SC. Human adenoviruses in water: occurrence and health implications: a critical review. Environ Sci Technol. 2006;40:7132-40. https:/ / doi.org/10.1021/es060892o

11. Rachmadi AT, Torrey JR, Kitajima M. Human polyomavirus: advantages and limitations as a humanspecific viral marker in aquatic environments. Water Res. 2016;105:456-69. https://doi.org/10.1016/j.watres.2016.09.010

12. Leone CM, Dharmasena M, Tang C, DiCaprio E, Ma Y, Araud E, et al. Prevalence of human noroviruses in commercial food establishment bathrooms. J Food Prot. 2018;81:719-28. https:// doi.org/10.4315/ 0362-028X.JFP-17-419

13. Kitajima M, Haramoto E, Phanuwan C, Katayama H. Prevalence and genetic diversity of Aichi viruses in wastewater and river water in Japan. Appl Environ Microbiol. 2011;77:2184-7. https:/ / doi.org/10.1128/ AEM.02328-10

14. Ganime AC, Leite JP, Figueiredo CE, Carvalho-Costa FA, Melgaço FG, Malta FC, et al. Dissemination of human adenoviruses and rotavirus species A on fomites of hospital pediatric units. Am J Infect Control. 2016;44:1411-3. https://doi.org/10.1016/j.ajic.2016.04.207

15. Ganime AC, Carvalho-Costa FA, Santos M, Costa Filho R, Leite JP, Miagostovich MP. Viability of human adenovirus from hospital fomites. J Med Virol. 2014;86:2065-9. https:/ / doi.org/10.1002/jmv.23907

16. Oristo S, Rönnqvist $M$, Aho $M$, Sovijärvi A, Hannila-Handelberg T, Hörman A, et al. Contamination by norovirus and adenovirus on environmental surfaces and in hands of conscripts in two Finnish garrisons. Food Environ Virol. 2017;9:62-71. https:// doi.org/10.1007/ s12560-016-9262-4

17. Maunula L, Kaupke A, Vasickova P, Söderberg K, Kozyra I, Lazic S, et al. Tracing enteric viruses in the 
European berry fruit supply chain. Int J Food Microbiol. 2013;167:177-85. https://doi.org/10.1016/j.ijfoodmicro. 2013.09.003

18. Dutilh BE, Cassman N, McNair K, Sanchez SE, Silva GG, Boling L, et al. A highly abundant bacteriophage discovered in the unknown sequences of human faecal metagenomes. Nat Commun. 2014;5:4498. https:// doi.org/10.1038/ ncomms5498

19. Guerin E, Shkoporov A, Stockdale SR, Clooney AG, Ryan FJ, Sutton TDS, et al. Biology and taxonomy of crAss-like bacteriophages, the most abundant virus in the human gut. Cell Host Microbe. 2018;24:653-664.e6. https:/ / doi.org/ 10.1016/j.chom.2018.10.002

20. Shkoporov AN, Khokhlova EV, Fitzgerald CB, Stockdale SR, Draper LA, Ross RP, et al. ФCrAss001 represents the most abundant bacteriophage family in the human gut and infects Bacteroides intestinalis. Nat Commun. 2018;9:4781. https:/ / doi.org/10.1038/s41467-018-07225-7

21. Stachler E, Kelty C, Sivaganesan M, Li X, Bibby K, Shanks OC. Quantitative CrAssphage PCR assays for human fecal pollution measurement. Environ Sci Technol. 2017;51:9146-54. https:// doi.org/10.1021/acs.est.7b02703

22. Stachler E, Akyon B, de Carvalho NA, Ference C, Bibby K. Correlation of crAssphage qPCR markers with culturable and molecular indicators of human fecal pollution in an impacted urban watershed. Environ Sci Technol. 2018;52:7505-12. https:// doi.org/10.1021/acs.est.8b00638

23. García-Aljaro C, Ballesté E, Muniesa M, Jofre J. Determination of crAssphage in water samples and applicability for tracking human faecal pollution. Microb Biotechnol. 2017;10:1775-80. https://doi.org/10.1111/1751-7915.12841

24. Ahmed W, Payyappat S, Cassidy M, Besley C, Power K. Novel crAssphage marker genes ascertain sewage pollution in a recreational lake receiving urban stormwater runoff. Water Res. 2018;145:769-78. https://doi.org/10.1016/ j.watres.2018.08.049

25. Ahmed W, Lobos A, Senkbeil J, Peraud J, Gallard J, Harwood VJ. Evaluation of the novel crAssphage marker for sewage pollution tracking in storm drain outfalls in Tampa, Florida. Water Res. 2018;131:142-50. https:/ / doi.org/ 10.1016/j.watres.2017.12.011

26. Park GW, Williamson KJ, DeBess E, Cieslak PR, Gregoricus N, De Nardo E, et al. High hand contamination rates during norovirus outbreaks in long-term care facilities. Infect Control Hosp Epidemiol. 2018;39:219-21. https://doi.org/10.1017/ice.2017.274

27. Cardemil CV, Kambhampati A, Grytdal S, Rodriguez-Barradas MC, Vargas B, Beenhouwer DO, et al. Incidence of norovirus and rotavirus from multisite active surveillance in Veteran's Affairs hospitals, December 2016-February 2018: results from the SUPERNOVA network. Open Forum Infect Dis. 2018;5(Suppl 1):S49. https://doi.org/10.1093/ofid/ofy209.118

28. Payne DC, Staat MA, Edwards KM, Szilagyi PG, Gentsch JR, Stockman LJ, et al. Active, population-based surveillance for severe rotavirus gastroenteritis in children in the United States. Pediatrics. 2008;122:1235-43. https://doi.org/10.1542/peds.2007-3378

29. Park GW, Chhabra P, Vinjé J. Swab sampling method for the detection of human norovirus on surfaces. J Vis Exp. 2017;120. https://doi.org/10.3791/55205
30. Liang Y, Jin X, Huang Y, Chen S. Development and application of a real-time polymerase chain reaction assay for detection of a novel gut bacteriophage (crAssphage). J Med Virol. 2018;90:4648. https:/ / doi.org/ 10.1002/jmv.24974

31. Montmayeur AM, Ng TF, Schmidt A, Zhao K, Magaña L, Iber J, et al. High-throughput next-generation sequencing of polioviruses. J Clin Microbiol. 2017;55:606-15. https://doi.org/10.1128/JCM.02121-16

32. Edgar RC. MUSCLE: multiple sequence alignment with high accuracy and high throughput. Nucleic Acids Res. 2004;32:1792-7. https://doi.org/10.1093/nar/gkh340

33. Guindon S, Dufayard JF, Lefort V, Anisimova M, Hordijk W, Gascuel O. New algorithms and methods to estimate maximum-likelihood phylogenies: assessing the performance of PhyML 3.0. Syst Biol. 2010;59:307-21. https:// doi.org/10.1093/sysbio/syq010

34. Lefort V, Longueville JE, Gascuel O. SMS: Smart Model Selection in PhyML. Mol Biol Evol. 2017;34:2422-4. https://doi.org/10.1093/molbev/msx149

35. Muhire BM, Varsani A, Martin DP. SDT: a virus classification tool based on pairwise sequence alignment and identity calculation. PLoS One. 2014;9:e108277. https://doi.org/10.1371/journal.pone.0108277

36. Trevethan R. Sensitivity, specificity, and predictive values: foundations, pliabilities, and piftalls in research and practice. Front Public Health. 2017;5:1-7. https://doi.org/10.3389/ fpubh.2017.00307

37. Wittkowski KM, Song T. Nonparametric methods for molecular biology. Methods Mol Biol. 2010;620:105-53. https://doi.org/10.1007/978-1-60761-580-4_2

38. Edwards RA, Vega AA, Norman HM, Ohaeri M, Levi K, Dinsdale EA, et al. Global phylogeography and ancient evolution of the widespread human gut virus crAssphage. Nat Microbiol. 2019;4:1727-36. https:// doi.org/10.1038/ s41564-019-0494-6

39. Cinek O, Mazankova K, Kramna L, Odeh R, Alassaf A, Ibekwe MU, et al. Quantitative CrAssphage real-time PCR assay derived from data of multiple geographically distant populations. J Med Virol. 2018;90:767-71. https://doi.org/ 10.1002/jmv.25012

40. Kirby AE, Streby A, Moe CL. Vomiting as a symptom and transmission risk in norovirus illness: evidence from human challenge studies. PLoS One. 2016;11:e0143759. https://doi.org/10.1371/journal.pone.0143759

41. Verani M, Bigazzi R, Carducci A. Viral contamination of aerosol and surfaces through toilet use in health care and other settings. Am J Infect Control. 2014;42:758-62. https://doi.org/10.1016/j.ajic.2014.03.026

42. Barker J, Jones MV. The potential spread of infection caused by aerosol contamination of surfaces after flushing a domestic toilet. J Appl Microbiol. 2005;99:339-47. https://doi.org/10.1111/j.1365-2672.2005.02610.x

43. Liang YY, Zhang W, Tong YG, Chen SP. crAssphage is not associated with diarrhoea and has high genetic diversity. Epidemiol Infect. 2016;144:3549-53. https:/ / doi.org/ $10.1017 /$ S095026881600176X

Address for correspondence: Geun Woo Park, Centers for Disease Control and Prevention, 1600 Clifton Rd NE, Mailstop H18-7, Atlanta, GA 30329-4027 USA; email: gpark@cdc.gov 